\title{
Clinical profile and microbiological trends of therapeutic keratoplasty at a network of tertiary care ophthalmology centers in India
}

\author{
Anthony Vipin Das · Sunita Chaurasia $\cdot$ Joveeta Joseph $\cdot$ Aravind Roy • \\ Sujata Das $\cdot$ Merle Fernandes
}

Received: 28 April 2021 / Accepted: 12 November 2021/Published online: 25 November 2021

(C) The Author(s), under exclusive licence to Springer Nature B.V. 2021

\begin{abstract}
Objective To describe the clinical profile and microbiology trends in patients undergoing therapeutic keratoplasty at a multi-tier ophthalmology hospital network in India.

Methods This cross-sectional hospital-based study included 3147 eyes undergoing therapeutic keratoplasty between Jan 2016 and Dec 2020 (5 years period) as cases. The demographic data, clinical
\end{abstract}

\section{A. V. Das}

Department of eyeSmart EMR and AEye, L V Prasad Eye Institute, Hyderabad, Telangana, India

S. Chaurasia $(\bowtie)$

Cornea and Anterior Segment Services, L V Prasad Eye Institute, Hyderabad 500034, Telangana, India

e-mail: sunita@lvpei.org

J. Joseph

Jhaveri Microbiology Center, Brien Holden Eye Research

Center, L. V. Prasad Eye Institute, Hyderabad,

Telangana, India

\section{A. Roy}

Kode Venkatadri Chowdary Campus, L V Prasad Eye

Institute, Vijayawada, India

S. Das

Mithu Tulsi Chanrai Campus, L V Prasad Eye Institute,

Bhubaneswar, Odisha, India profile and microbiological analysis were collected using an electronic medical record system.

Results Of a total of 13,625 eyes with microbial keratitis(non-viral), 3147 (23.1\%) underwent therapeutic keratoplasty during the study duration. Majority of patients were males $(68.35 \%)$, from a rural geography $(49.89 \%)$ and in 51-60 years age bracket $(23.74 \%)$. The mean age of the patients was $50.54 \pm 15.83$ years. Two-thirds of patients were from lower socio-economic strata $(66.63 \%)$ with an

\author{
M. Fernandes \\ Grandhi Mallikarjun Rao Varalakshmi Campus, L V \\ Prasad Eye Institute, Vishakhapatnam, \\ Andhra Pradesh, India
}


agrarian background (36.51\%). Of the 3,147 eyes, fungus $(51.8 \%)$ was the most common indication of therapeutic keratoplasty followed by bacteria $(16.87 \%)$ and parasite $(1.27 \%)$. No organisms could be identified in about a third $(29.33 \%)$ of the cases. Between 2016 and 2020, the trend of therapeutic keratoplasties for fungal infections steadily grew (39.9\% vs $45.49 \%)$ while the bacterial infections showed a steady decline $(23.15 \%$ vs $11.81 \%)$.

Conclusions Medical cure rate was seen in majority of those with microbial keratitis, and $23.1 \%$ eventually required management with therapeutic keratoplasty. Fungal keratitis was the most common indication for therapeutic keratoplasty. Male gender, rural setting, low socio-economic background and agricultural occupation are common risk factors for patients undergoing therapeutic keratoplasty in India.

Keywords Therapeutic keratoplasty · Keratoplasty · Keratitis · India $\cdot$ Electronic medical records

\section{Introduction}

Microbial keratitis substantially contributes to global blindness and is estimated to be the fourth leading cause of blindness worldwide [1]. Medical treatment is the first line of management and can be effective in achieving a clinical cure when specific treatment is instituted at an early stage of the disease. Those with advanced disease and refractory to the medical treatment are managed by performing therapeutic keratoplasty. The primary aim of the surgery is to eliminate the infective foci and restore globe integrity [2]. The procedure involves a meticulous surgery, intensive post-operative management care and close follow up for complications particularly recurrence of infection in cases due to fungus and parasitic infection.

There is a distinct geographic variation in the burden of microbial keratitis. The countries with developed health care system report lower number of cases requiring therapeutic keratoplasty [3-5] compared to the ones with a developing health care system, where it contributes to a significant percentage of all keratoplasties [6-8]. Severity of the infection, delayed presentation and empirical treatment due to lack of microbiological confirmation have all been shown to contribute to poor outcomes in patients with microbial keratitis [5-7, 9].

Knowledge about the evolving trends in the microbiological spectrum of the causative organisms is crucial to help better manage care in these patients. The initial pre-operative microbiological work up from the corneal scraping samples, the cultures and histology of the host corneal button excised during therapeutic keratoplasty provide invaluable information that guide the medical therapy in the postoperative period, to eliminate the infection and achieve a clinical cure [10].

There is a paucity of large series on the clinical profile and microbiological trends of therapeutic keratoplasty in the Indian population. The aim of the authors in this study is to present the clinical profile and the microbiological trends of patients undergoing therapeutic keratoplasty at a large multi-tier ophthalmology network in India using electronic medical record driven analytics.

\section{Materials and methods}

Study design, period, location and approval

This cross-sectional observational hospital-based study included all patients undergoing therapeutic keratoplasty between January 2016 and December 2020 to the tertiary centers of an ophthalmology network spread across 4 adjacent neighboring states (Telangana, Andhra Pradesh, Odisha and Karnataka) of India [11]. A standard consent form for electronic data privacy was filled by the patient or the parents or guardians of the patient at the time of registration. None of the data that were used for analysis had identifiable parameters of the patient. The study adhered to the Declaration of HELSINKI and was approved by the Institutional Ethics Committee, Hyderabad, India. The clinical data of each patient who underwent a comprehensive ophthalmic examination using a standardized template were entered into a browser-based electronic medical records system (eyeSmart EMR) by uniformly trained ophthalmic personnel and supervised by an ophthalmologist [12].

The standard protocol of management of microbial keratitis across the network is described in a previous study from the institute [7]. In brief, all eyes diagnosed to have keratitis on their first visit to the clinic, 
irrespective of their past diagnosis and treatment, undergo a routine microbiological evaluation that involves smear and culture examination of samples obtained on corneal scrapings. If the keratitis deteriorates (defined as increase in size of the infiltrate and exudates in the anterior chamber and/ or large perforation), therapeutic keratoplasty is planned on an emergency basis utilizing donor corneas provided by the network of eye banks affiliated to the institute $[13,14]$. At the time of therapeutic keratoplasty (whenever possible), the infected corneal button is divided into two parts. One half of the button is sent for microbiological evaluation where it is further cut and inoculated onto culture media such as blood agar, sabouraud's dextrose agar, brain heart infusion and non-nutrient agar. The second half is sent in formalin $1 \%$ for routine histopathological analysis.

Cases

A total of 3147 eyes of patients of all ages underwent therapeutic keratoplasty at the four tertiary centers of the network during the study period. The eyeSmart EMR was initially screened for patients with: (i) completed operation coded as therapeutic keratoplasty (ii) and final microbiological diagnosis of microbial keratitis (non-viral) entered in the EMR. A total of 3,147 eyes were identified as cases, and the demographic data, clinical profile and microbiological analysis were extracted from the eyeSmart EMR database system.

Data retrieval and processing

The data of 3147 eyes included in this study were retrieved from the electronic medical record database and segregated in a single excel sheet. The columns included details such demographics, clinical presentation, surgical notes and confirmed microbiological diagnosis. The data from the excel sheet were extracted and used for analysis using the appropriate statistical software. Standardized definitions were used for occupation, socio-economic status and geographic categorization [15]. The visual acuity was classified according to the WHO guidelines [16].
Statistical analysis

Descriptive statistics using mean \pm standard deviation and median with Inter-quartile range (IQR) were used to elucidate the demographic and microbiological data. Microsoft Excel (Microsoft Corporation. 2018. Redmond, USA) was used for statistical analysis and graphs.

\section{Results}

Microbial keratitis trends

A total of 13,625 cases of Microbial (Non -Viral) Keratitis were diagnosed during the 5-year study period across the tertiary centers of the eye care network. Those with the diagnosis of herpes simplex virus (HSV) keratitis were excluded. There were 5954 (43.7\%) cases with fungal etiology, 3194 (23.44\%) cases were bacterial, $521(3.82 \%)$ cases with mixed infections, $213(1.56 \%)$ cases were parasitic, 3134 (23\%) cases yielded no organism on microbiological work up, and the information was not available (those with the diagnosis of microbial keratitis, but absence of tissue sample either corneal scrapings or corneal tissue in the microbiology and pathology laboratory) in $609(4.47 \%)$ cases (Table 1). The yearly trends of the microbial keratitis are presented in Fig. 1. Overall, 3,147 patients underwent therapeutic keratoplasty accounting for $23.1 \%$ of the microbial keratitis cases diagnosed in the same period.

Microbiological analysis and therapeutic keratoplasty trends

(Table 1) In the microbiological analysis of the corneal button of 3147 eyes that underwent therapeutic keratoplasty, fungus was identified in 1630 (51.8\%) eyes, bacteria in $531(16.87 \%)$ eyes, parasite (either Acanthamoeba/ Microsporidia) in 40 (1.27\%) eyes and no growth in $923(29.33 \%)$ eyes. Mixed infections were seen in $305(9.69 \%)$ patients, and there was no information available in a minority of $23(0.73 \%)$ patients. Over 2016-2020, the trends of the bacterial keratitis showed a steady decline $(23.15 \%$ vs. $11.81 \%$ ), while the trends of fungal keratitis showed a steady increase $(39.9 \%$ vs. $45.49 \%)$. The trends of keratitis with no growth on microbiology workup also 
Table 1 Table showing the number of eyes diagnosed with microbial keratitis, with clinical diagnosis and number of eyes requiring therapeutic keratoplasty (Th PK)

\begin{tabular}{lllllll}
\hline Parameters & Overall & Fungal & Bacterial & Parasitic & $\begin{array}{l}\text { No organisms on } \\
\text { microbiological workup }\end{array}$ & $\begin{array}{c}\text { Not available } \\
\text { (samples not available for } \\
\text { analysis) }\end{array}$ \\
\hline $\begin{array}{l}\text { No of eyes with microbial } \\
\text { keratitis }\end{array}$ & 13,625 & 5954 & 3194 & 213 & 3134 & 609 \\
$\begin{array}{l}\text { No of eyes that underwent } \\
\text { Th PK }\end{array}$ & 3147 & 1630 & 531 & 40 & 923 & 23 \\
$\begin{array}{l}\% \text { of eyes that needed Th } \\
\text { PK }\end{array}$ & 23.1 & 27.38 & 16.62 & 18.78 & 29.45 & 3.78 \\
\hline
\end{tabular}

Fig. 1 Trends of etiological organism in Microbial Keratitis (2016-2020)

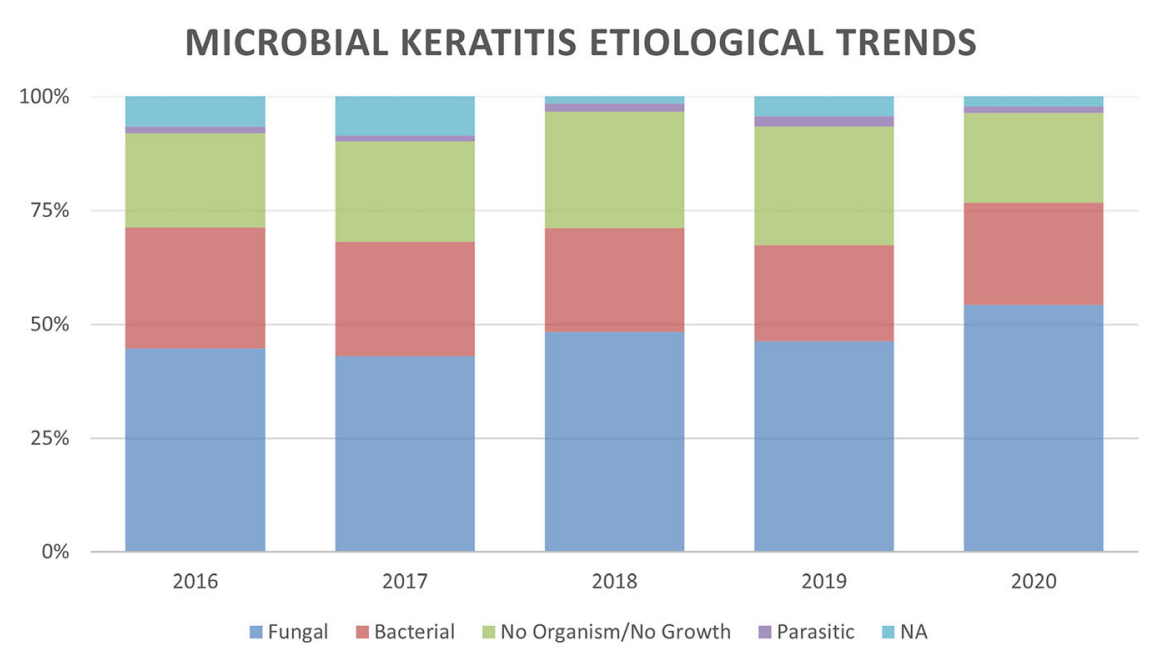

grew steadily from $24.63 \%$ vs. $35.24 \%$. The annual distribution of the microbiological profile is detailed in Fig. 2.

a. Fungal overview: In the eyes documented with fungal growth, Fusarium species was identified in $573(35.15 \%)$ eyes, Aspergillus species in 564 (34.6\%) eyes, Pythium insidiosum in 104 (6.38\%) eyes, other hyaline fungus in $98(6.01 \%)$ eyes, dematiaceous fungus in 96 (5.89\%) eyes, Candida species in $22(1.35 \%)$ eyes and miscellaneous species were identified in 173 (10.61\%) eyes.

b. Bacterial and parasitic overview: In the eyes documented with bacterial growth that included mixed infections, gram-positive cocci were identified in 453 (54.19\%) eyes, gram negative bacilli in $291(34.81 \%)$ eyes and gram-positive bacilli in
$92(11 \%)$ eyes. Among the eyes documented with parasitic growth, Acanthamoeba was responsible for the $37(1.27 \%)$ and microsporidia in 3 eyes.

Age

The mean age of the patients who underwent therapeutic keratoplasty was $50.54 \pm 15.83$ years while the median age was 51 (IQR: 40-62) years. The overall distribution was $2.76 \%(87 / 3,147)$ in children $(\leq 16$ years $)$ and $97.24 \%(3,060 / 3,147)$ in adults (above 16 years). The frequency distribution of therapeutic keratoplasty showed an increase between 31 and 40 years of age $(15.25 \%)$ and peaked between 51 and 60 years of age $(23.74 \%)$ followed by gradual decline from 61 to 70 years of age $(20.21 \%)$ in the 
Fig. 2 Microbiological trends in therapeutic keratoplasty (2016-2020)
MICROBIOLOGICAL TRENDS OF THERAPEUTIC KERATOPLASTY

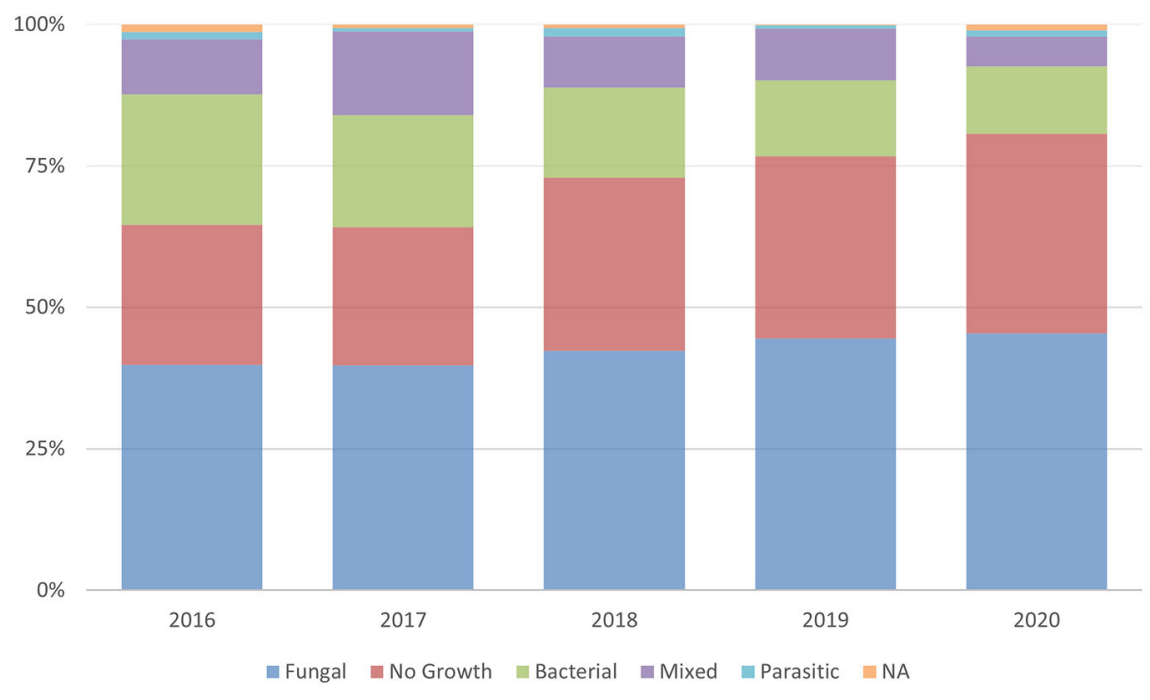

subsequent decades thereafter. The decade wise distribution of the patients is detailed in Fig. 3.

Sex

There were $2151(68.35 \%)$ male and 996 (31.65\%) female patients who underwent therapeutic keratoplasty. Among the patients, the mean and median age were $51.37 \pm 15.67$ and 53 (IQR: 41 to 63 ) years for men and $48.76 \pm 16.01$ and 50 (IQR: 38 to 61 ) years for women, respectively. The overall mode was 56 years; 56 years for men and 51 years for women.

\section{Rural-urban-metropolitan distribution}

There were 1570 (49.89\%) patients undergoing therapeutic keratoplasty from rural districts, 1483 (47.12\%) from urban districts and 94 (2.99\%) from metropolitan regions.

\section{Socio-economic status}

There were 2097 (66.63\%) patients undergoing therapeutic keratoplasty from the lower socio-economic class, 984 (31.27\%) from the lower middle class, 54 $(1.72 \%)$ from the upper middle class and $12(0.38 \%)$ from the upper class. The overall frequency distribution was significantly higher $(p<0.00001)$ in the lower socio-economic class as compared to the other classes.

\section{Occupation}

Of the 3147 patients who underwent therapeutic keratoplasty, 1149 (36.51\%) were agriculture related; 556 (17.67\%) were homemakers; 534 (16.97\%) were professionals; 499 (15.86\%) were manual laborers; $262(8.33 \%)$ were currently not employed (retired or unemployed); 108 (3.43\%) were students; and in the remaining $39(1.24 \%)$ the occupational category was not available/applicable.

Laterality and year of surgery

The left eye was operated in $1611(51.19 \%)$ cases and the right eye in 1536 (48.81\%) cases. An average of 629 therapeutic keratoplasties were performed each year during 2016-2020 with the highest of 662 $(21.04 \%)$ in the year 2017 to the lowest of 576 $(18.3 \%)$ in the year 2020. The year wise distribution of the patients is detailed in Fig. 4.

Risk factors

The concomitant risk factors documented at the time of first visit were diabetes in $226(7.18 \%)$, chronic 
Fig. 3 Decade wise distribution of patients who underwent therapeutic keratoplasty (2016-2020)

\section{Decade wise distribution of Therapeutic Keratoplasty}

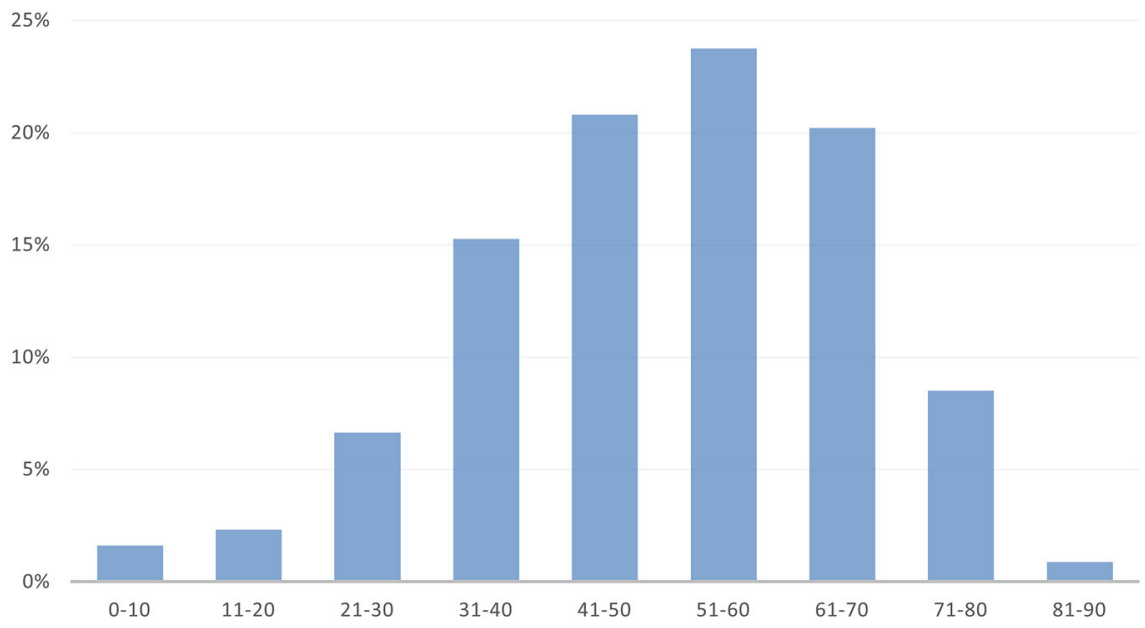

alcoholism in 118(3.75\%), smoking in 97(3.08\%), history of contact lens usage in 10(0.32\%), HIV infection in $2(0.06 \%)$ and prior usage of steroids in $46(1.46 \%)$ patients.

Presenting visual acuity

In the 3147 patients undergoing therapeutic keratoplasty, mild or no visual impairment (20/20 to 20/70) was seen in $5(0.16 \%)$ patients, moderate visual impairment $(>20 / 70$ to $20 / 200)$ in $14(0.44 \%)$ patients, severe visual impairment $(>20 / 200$ to 20/400) in $8(0.25 \%)$ patients, blindness $3(>20 / 400$ to $20 / 1200)$ in $1,307(41.53 \%)$ patients, blindness 4 ( $>20 / 1200$ to Perception of light) in 1,399 (44.46\%) patients and blindness 5 (No perception of light) in 9 $(0.29 \%)$ patients. The visual acuity was undetermined or unspecified in 405 (12.87\%) patients. The 9 patients who had no light perception underwent keratoplasty for tectonic reasons.

\section{Discussion}

This study sought to describe the clinical profile and the microbiological trends of patients undergoing therapeutic keratoplasty at a large multi-tier ophthalmology network in India using electronic medical record driven analytics. An important aspect of the study was to determine the trend of the microbial infection between 2016 and 2020 (5 years period) that eventually necessitates therapeutic keratoplasty. An understanding of microbiological trend over the years helps to understand the changing profile of the causative organisms of microbial keratitis requiring therapeutic keratoplasty.

The subset of patients with microbial keratitis that need therapeutic keratoplasty range from 3.3 to $38 \%$ in different studies that vary in sample size, geographical locations and microbiological spectrum [17]. The Asia Cornea Society Infectious Keratitis Study [18], that included one of our centers, reported 9.47\% (628/ 6626) eyes that were managed by therapeutic keratoplasty. Based on EMR analytics, in the present study we screened the largest number of patients with microbial keratitis that were treated across the network of tertiary care centers. Majority of microbial keratitis patients received medical treatment alone, and surgical intervention via therapeutic keratoplasty was required in $23.1 \%$ of the patients with keratitis. Although several factors determine the medical cure rate in microbial keratitis using anti-biotic therapy alone, the observation that less than one-quarter patients were managed via therapeutic keratoplasty indirectly suggests a good medical cure rate with the available anti-biotic modalities of treatment.

The Asia Cornea Society Infectious Keratitis Study published in 2018, included data from 8 countries 
Fig. 4 Year wise distribution of therapeutic keratoplasty (2016-2020)
Yearly Trend of Therapeutic Keratoplasty

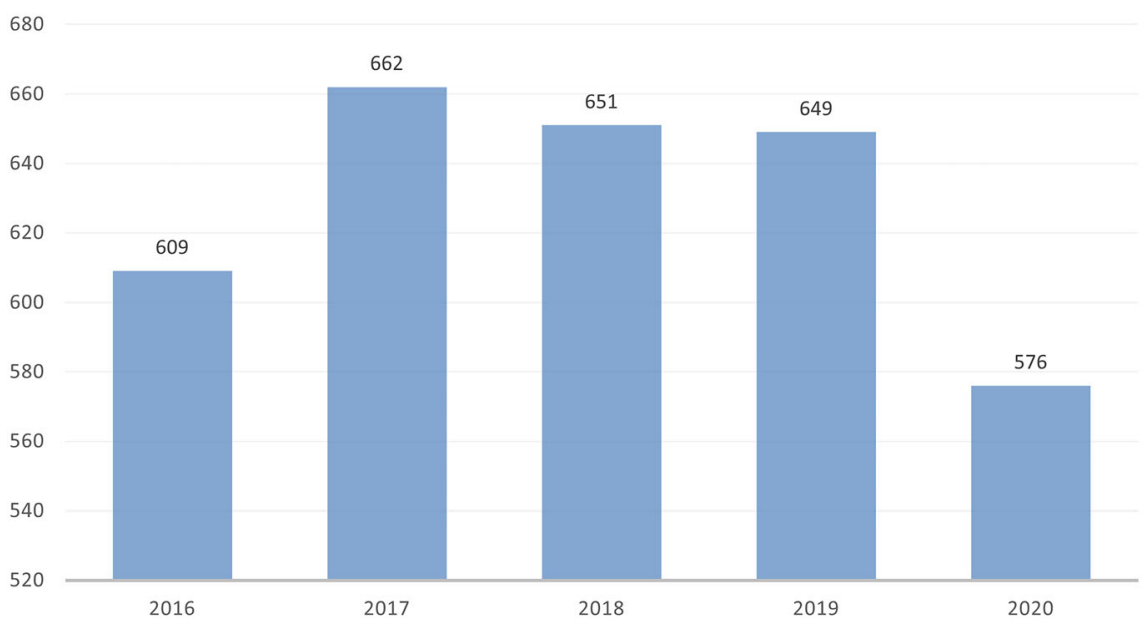

(India, China, Japan, South Korea, Taiwan, Thailand, the Philippines and Singapore) and reported country specific differences. ${ }^{18}$ Bacterial keratitis was the most common diagnosis in South Korea (42.8\%) and Singapore $(41.3 \%)$. Fungal species were by far the most common microorganisms isolated in China and India, with Fusarium species being the most common pathogen in China (30.9\%) and India (25.7\%). In the remaining countries, the most common microorganism isolated were typically bacterial, such as $\mathrm{Pseu}$ domonas aeruginosa in the Philippines (12.5\%), Taiwan $(31.9 \%)$, Thailand $(32.1 \%)$ and Singapore (42.2\%), and Propionibacterium acnes in Japan $(33.0 \%)$ and South Korea (10.8\%). In this study, fungal keratitis $(5964 / 13,625 ; 43.7 \%)$ was the commonest cause of microbial keratitis, followed by bacterial $(31,194 / 13625 ; 23.44 \%)$ and parasitic $(213 /$ $13625 ; 1.56 \%$ ) keratitis. In our cohort of patients, over 2016-2020, the trend of fungal infections steadily grew (39.9\% vs $45.49 \%$ ) while the bacterial infections showed a steady decline ( $23.15 \%$ vs. $11.81 \%$ ).

A study from Taiwan by Fung-Rong $\mathrm{Hu}$ et al. described their experience of 107 therapeutic keratoplasties and the microbiological diagnosis [8]. They reported bacteria to be the most common cause with gram negative organisms accounting for the majority $(51.6 \%)$ of the cases followed by gram-positive organisms (41.9\%) for the rest of the cases. In this study, the most common indication for therapeutic keratoplasty was fungal keratitis $(51.8 \%)$ in over half of the cases followed by bacteria (16.87\%). The most common fungal species identified were Fusarium (35.15\%) and Aspergillus (34.6\%) each accounting for a third of the fungal cases. Among bacterial causes of therapeutic keratoplasty, we found gram-positive cocci accounted for the majority $(54.19 \%)$ followed by gram negative bacilli (34.81\%) and gram-positive bacilli $(11 \%)$. We also noted an increasing trend of culture negative microbial keratitis requiring therapeutic keratoplasty (24.63\% in 2016 vs. $35.24 \%$ in 2020). The culture negative results are best explained by either a combination of medications started empirically prior to presentation to a tertiary eye care center, prolonged and varied therapy with delayed presentation and inadequate number of organisms for culture from the sample taken. Additionally, those eyes with near total corneal melt/perforation may have had little yield for microbiological culture studies and may have had the final diagnosis of microbial keratitis based on either clinical impression or smear examination. The increase in this trend is likely related to COVID-19 situation in the year 2020 [14]. As both the clinical and paraclinical services (Microbiology laboratory) had to work with reduced work force and there were travel restrictions, it is possible that only limited number of samples were received and tested in the microbiology laboratory services at individual centers.

In this cohort, the most common systemic risk factor identified was diabetes mellitus. A history of contact lens usage was noted in a small percentage 
$(<0.32 \%)$. Males accounted for a higher percentage $(68.35 \%)$ and the agrarian occupation which is common in India accounted for a third of the patients (36.51\%) who underwent therapeutic keratoplasty in our cohort. There is a need to design and implement knowledge, awareness and prevention programs to address this vulnerable group susceptible to microbial keratitis that subsequently adds to the burden of therapeutic keratoplasty.

The greatest strengths of the study include the large sample size of the cohort and a complete electronic medical record entry of the parameters that enabled efficient documentation and analysis. The eye care network is also equipped with an experienced microbiology service and clinical protocols that document corneal scraping for every suspected microbial keratitis and corneal button culture and identification of the organism post therapeutic keratoplasty. This represents real world data on a large series of microbial keratitis requiring therapeutic keratoplasty. The limitations of the study, however include its retrospective nature and the maximum medical therapy threshold at which surgical intervention would have been planned in the patients.

In conclusion, this analysis of a vast number of therapeutic keratoplasties performed in a multi-tier ophthalmology network revealed that fungal keratitis as the main indication was on the rise particularly in males with an agrarian background hailing from rural areas and resulted in blindness in almost half the cohort. Hence, there is an unmet need for community programs targeting these vulnerable groups to prevent this sight threatening infection, reduce the need for therapeutic keratoplasty and its associated visual morbidity.

Acknowledgements The authors acknowledge the support of Department of eyeSmart EMR \& AEye team specially Mr. Ranganath Vadapalli and Mr. Mohammad Pasha, Ocular pathology laboratory and Microbiology services affiliated to LV Prasad Eye Institute.

Author contributions The corresponding author states that authorship credit of this manuscript was based on (1) substantial contributions to conception and design, acquisition of data or analysis and interpretation of data; (2) drafting the article or revising it critically for important intellectual content; and (3) final approval of the version to be published. All listed authors met conditions 1, 2 and 3. All persons designated as authors qualify for authorship, and all those who qualify are listed. Each author has participated sufficiently in the work to take public responsibility for appropriate portions of the content.
Funding Hyderabad Eye Research Foundation, Hyderabad, India.

\section{Declarations}

Conflicts of interest The authors declare that they do not have any conflict of interest.

Ethics approval This study involving human participants was in accordance with the ethical standards of the institutional and national research committee and with the 1964 Declaration of HELSINKI and its later amendments or comparable ethical standards. The Institutional Ethics Committee of L V Prasad Eye Institute, Hyderabad, India approved this study.

Informed consent Informed consent was obtained from all individual participants included in the study.

\section{References}

1. Pascolini D, Mariotti SP (2012) Global estimates of visual impairment: 2010. Br J Ophthalmol 96(5):614-618

2. Fine M (1960) Therapeutic keratoplasty. Trans Acad Ophthalmol Otolaryngol 64:786-808

3. Benson MD, Kurji K, Tseng C, Bao B, Mah D (2018) Analysis of penetrating keratoplasty in Northern Alberta, Canada, from 2000 to 2015. Can J Ophthalmol 53(6):568-573

4. Nielsen SE, Nielsen E, Julian HO, Lindegaard J, Højgaard K, Ivarsen A, Hjortdal J, Heegaard S (2015) Incidence and clinical characteristics of fungal keratitis in a Danish population from 2000 to 2013. Acta Ophthalmol 93(1):54-58

5. Nowik KE, Wylęgała A, Nowik K, Wylęgała E (2020) A single-centre retrospective observational study of fungal keratitis in Poland with a review of findings in Europe. Ann Agric Environ Med 27(3):343-347

6. Sharma N, Jain M, Sehra SV, Maharana P, Agarwal T, Satpathy G, Vajpayee RB (2014) Outcomes of therapeutic penetrating keratoplasty from a tertiary eye care centre in northern India. Cornea 33(2):114-118

7. Mundra J, Dhakal R, Mohamed A, Jha G, Joseph J, Chaurasia S, Murthy S (2019) Outcomes of therapeutic penetrating keratoplasty in 198 eyes with fungal keratitis. Indian J Ophthalmol 67(10):1599-1605

8. Tew TB, Chu HS, Hou YC, Chen WL, Wang IJ, Hu FR (2020) Therapeutic penetrating keratoplasty for microbial keratitis in Taiwan from 2001 to 2014. J Formos Med Assoc 119(6):1061-1069

9. Shah H, Radhakrishnan N, Ramsewak S, Chiu S, Joseph S, Rose-Nussbaumer J, Prajna NV (2019) Demographic and socioeconomic barriers and treatment seeking behaviors of patients with infectious keratitis requiring therapeutic penetrating keratoplasty. Indian J Ophthalmol 67(10):1593-1598

10. Das S, Sharma S, Priyadarshini O, Sahu SK, Kar S, Vemuganti GK (2011) Association between culture results of corneal scrapings and culture and histopathology results of corneal tissues in therapeutic keratoplasty. Cornea 30(9):1003-1006 
11. Rao GN, Khanna RC, Athota SM, Rajshekar V, Rani PK (2012) Integrated model of primary and secondary eye care for underserved rural areas: The L V Prasad Eye Institute experience. Indian J Ophthalmol 60:396-400

12. Das AV, Kammari P, Vadapalli R, Basu S (2020) Big data and the eyeSmart electronic medical record system - An 8 -year experience from a three-tier eye care network in India. Indian J Ophthalmol 68(3):427-432

13. Chaurasia S, Mohamed A, Garg P, Balasubramanian D, Rao GN (2020) Thirty years of eye bank experience at a single centre in India. Int Ophthalmol 40(1):81-88

14. Roy A, Das S, Chaurasia S, Fernandes M, Murthy S (2020) Corneal transplantation and eye banking practices during COVID-19-related lockdown period in India from a network of tertiary eye care centers. Indian J Ophthalmol 68(11):2368-2371

15. Das AV, Podila S, Prashanthi GS, Basu S (2020) Clinical profile of pterygium in patients seeking eye care in India: electronic medical records-driven big data analytics report III. Int Ophthalmol 40(6):1553-1563
16. World Health Organization. (2008). Change the Definition of Blindness [PDF file]. Retrieved from https://www.who. int/blindness/Change \%20the\%20Definition\%20of\% 20Blindness.pdf

17. Sharma N, Sachdeva R, Jhanji V, Titiyal JS, Vajpayee RB (2010) Therapeutic keratoplasty for microbial keratitis. Curr Opin Ophthalmol 21:293-300

18. Khor WB, Prajna VN, Garg P, Mehta JS, Xie L, Liu Z, Padilla MDB, Joo CK, Inoue Y, Goseyarakwong P, Hu FR, Nishida K, Kinoshita S, Puangsricharern V, Tan AL, Beuerman R, Young A, Sharma N, Haaland B, Mah FS, Tu EY, Stapleton FJ, Abbott RL, Tan DT; ACSIKS Group (2018) The Asia Cornea Society Infectious Keratitis Study: A Prospective Multicenter Study of Infectious Keratitis in Asia. Am J Ophthalmol 195:161-170

Publisher's Note Springer Nature remains neutral with regard to jurisdictional claims in published maps and institutional affiliations. 